\title{
The molecular defect underlying canine fucosidosis
}

\author{
Barbara J Skelly, David R Sargan, Michael E Herrtage, Bryan G Winchester
}

\begin{abstract}
Fucosidosis is a lysosomal storage disease which affects humans and English springer spaniel dogs. The disease is recessively inherited in both species and results from a deficiency of the enzyme $\alpha-L-$ fucosidase. We have recently cloned and sequenced the canine fucosidase gene (EMBL sequence admission number X92448 (cDNA) and X92671-X92678 (individual exonic data)). The gene spans $12 \mathrm{~kb}$ and consists of eight exons. SSCP based mutation analysis of affected animals was carried out on the coding region of this gene both with exonic primers, and intronic primer pairs for each exon. A 14 base pair deletion of the cDNA was identified at the $3^{\prime}$ end of exon 1 in fucosidosis affected animals. Surprisingly, PCR based genomic cloning of DNA from these animals showed an identical deletion in this DNA, ending at the start of intron 1. This change causes a frameshift and, in consequence, 25 novel codons are transcribed in exon 2 before the first of two adjacent premature stop codons is encountered.

(f Med Genet 1996;33:284-288)
\end{abstract}

Key words: canine fucosidosis; $\alpha$-L-fucosidase; lysosomal storage disease; mutation detection.

The lysosomal storage disease fucosidosis has been described in humans ${ }^{1}$ and also in pedigree English springer spaniels in Australia and in the United Kingdom. ${ }^{23}$ Affected dogs have a profound deficiency $(0-5 \%$ of the control mean) of the enzyme $\alpha$-L-fucosidase which catalyses removal of fucose moieties from the oligosaccharide side chains of glycoproteins and glycolipids. Pathology and clinical signs are the result of the lysosomal accumulation of fucose containing glycoconjugates. ${ }^{4}$ The central nervous system displays particular marked storage abnormalities. Heterozygotes are without symptoms and possess fucosidase activities of approximately $50 \%$ of the control mean. The disease in the springer spaniel manifests as a mainly neurological syndrome with mixed motor and mental function deficits including progressive ataxia, proprioceptive difficulties, apparent blindness, change in temperament, dysphagia, dysphonia, and loss of learned behaviour. ${ }^{5}$ As the disease progresses there is a rapid deterioration in body condition with muscle wasting. Affected dogs die or are euthanased by 3 to 4 years of age. The canine disease has been likened to the delayed onset human form because dogs are clinically normal until 18 months of age. Thus, disease similarities have led to the use of springer spaniel dogs as models in bone marrow transplantation trials. $^{67}$

Both the canine and the human diseases are inherited as autosomal recessive conditions. Pedigree analysis of canine families suggests that a common British founder is present in all affected lines, including those in Australia which were derived from exported British breeding stock. Testing for carrier status in dogs has been conducted in the UK by analysis of enzyme activity. ${ }^{8}$ Heterozygotes should have enzyme activities that are approximately $50 \%$ of the control mean. The division between normal and heterozygous dogs is, however, often indistinct and a molecular description of the disease causing mutation would be valuable in allowing definitive designations of genotype within the springer spaniel population.

The cDNA for human $\alpha$-L-fucosidase (FUCA1) has been cloned, ${ }^{9}$ and the genomic organisation established. ${ }^{10}$ In addition, most families with affected family members have had the molecular basis of the disease investigated. Early analyses described the obliteration of an $E c o$ RI site in six patients from five fucosidosis families $^{112}$ and the deletion of the last two exons at the $3^{\prime}$ end of the gene. ${ }^{13}$ As more patients are analysed, it has become clear that there is considerable mutational variety within the patient groups studied. ${ }^{14-19}$ Most of the mutations described involve single base substitutions or small deletions or insertions. One splice site mutation has been identified. ${ }^{14} \mathrm{No}$ obvious mutation "hotspots" have so far been identified in human fucosidosis.

We have recently cloned and sequenced the canine gene for fucosidase (Skelly et al, submitted for publication). The nucleotide sequence data used in this paper will appear in the EMBL, Genbank, and DDBJ Nucleotide Sequence Databases under the accession numbers X92448 (cDNA sequence) and X92671X92678 (sequences of exons 1-8). In this study we have used SSCP and sequence analysis to investigate the mutation responsible for canine fucosidosis.

\section{Materials and methods}

The dogs investigated in this work were from pedigree English springer spaniel breeding colonies in the UK. Affected animals were identified by enzymology as previously described ${ }^{8}$ following referral to the Department of Clinical Veterinary Medicine, University of Cambridge, or were identified from samples submitted directly by veterinarians. 
A

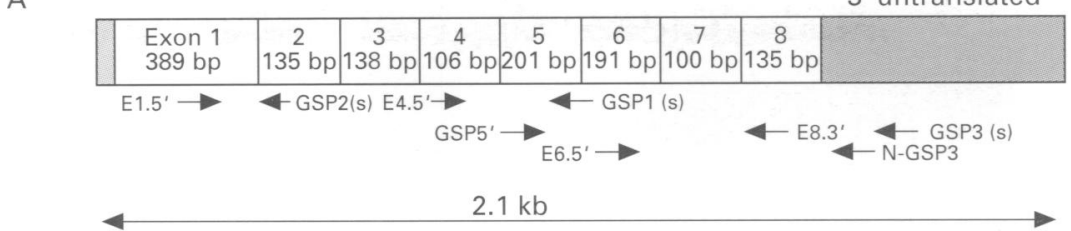

B

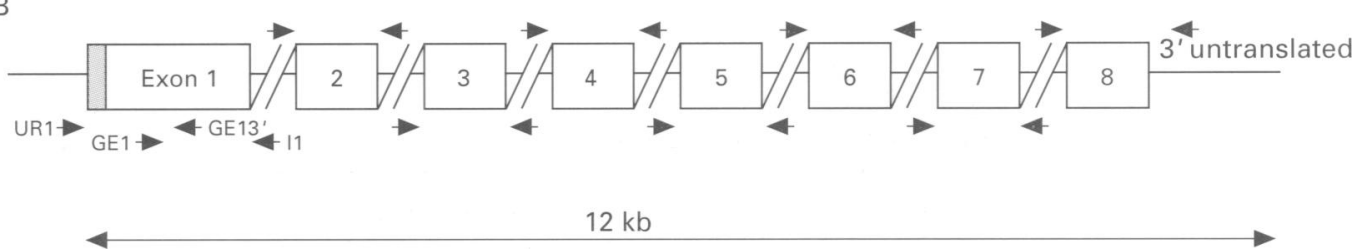

Figure 1 The strategy used for mutation analysis of the canine fucosidase cDNA and gene. ( $A$ ) The cDNA structure showing the positions of primers used in RT-PCR analysis (denoted by arrows). Primers used for cDNA synthesis are marked with an s. The $5^{\prime}$ end of exon 1, part of exon 2, and exon 3 were not included in the RT-PCR analysis (see text for explanation of mutation analysis strategy). (B) The SSCP analysis was continued using intronic primer pairs for each exon individually. Exon 1 is the largest exon and was divided into two overlapping pieces for PCR and SSCP. The signal peptide was included in the amplification of the $5^{\prime}$ end of exon 1. This exon proved difficult to amplify owing to the high $G / C$ content of the exon and adjacent intron. In both diagrams the hatched $5^{\prime}$ area denotes the signal peptide.

SSCP ANALYSIS

SSCP analysis was performed across the whole gene on genomic DNA isolated from blood or from fibroblast cultures by the guanidinium hydrochloride method of extraction. ${ }^{20}$ SSCP analysis of fucosidase cDNA was also performed; however, the RT-PCR products generated did not span the whole of the coding region (figure 1A). Once intronic and slice site sequence information was available, mutation analysis of the exons and flanking regions using intronic primers was deemed preferable. PCR products for SSCP analysis were in all cases smaller than $350 \mathrm{bp}$. Genomic and RT-PCR products from normal and affected dogs were amplified as described below, denatured in formamide dye, and electrophoresed at room temperature in non-denaturing gels (Hydrolink MDE gel solution, AT Biochem) at 6 to $8 \mathrm{~W}$ for 12 to 14 hours. $^{2122}$ For each exon or RTPCR product analysed, SSCP was performed both with and without the addition of $10 \%$ glycerol.

PCR AMPLIFICATION

For amplification before SSCP analysis of each exon plus the flanking regions, primers were designed in adjacent intronic sequences and

Primer sequences for SSCP analysis of canine fucosidase

Primer pairs for $c D N A$ analysis (5' primer precedes $\left.3^{\prime}\right)$

E1.5' GCGAGTGGTTCTGGTGGCAC GSP2 GGACCCACATCATTAGAGTTCCA

E4.5 TTTGGTCTGATGGGGAGTGG GSP1 CTGAACCAGTTCCGAGATGATTTCACATTC

GSP5" "GTTCCTGTCACCATGGAGGATACTACAAC E8.3' ACTGGGCAGATAAATGAGCAG

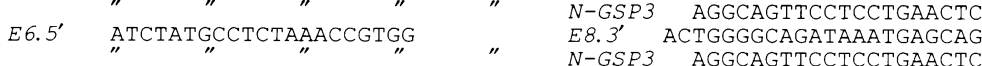

GSP3 TGGTGCTATAAGAGGAAACCAAAGCAGCC

Primers used for genomic DNA PCR analysis ( $5^{\prime}$ primer precedes $3^{\prime}$ )

Exon 1 5, URI GTACCTGCGCGGAGAGAGG

Exon 1 3' GE1 GCGAGTGGTTCTGGTGGCAC

Exon 2

Exon 3

Exon 4

Exon 5

Exon 6 TGCTTCCTGTGCTTATGTAG CTTTACTTAATGGACATTTGCT CCATGAGATATCACTGTGAAT GTAGTTTTACCCCAGATGTC TTGGCACTCGCAGCTAGGAT ATACTCAGGGTGTTGATGTC
I IACGTCCAGTGCGCACCCT GAACAGAGAGGAATGAAAGATC ACTGATCAAAGTCATAAGGGC TTCATACTCCAGATCTTGGC CTCTTAGTCAGAAGGCTAGT CGACAGTCCTGCTCCTACAT CTTTAGGAAGGTCACCTCTC
AGGCAGTTCCTCCTGAACTC were situated approximately 50 base pairs from the consensus sequences surrounding each splice site (fig $1 \mathrm{~B}$, table). For exons $2-8$ reactions were carried out using $0.5 \mathrm{U}$ of $\mathrm{Taq}$ polymerase (Gibco-BRL) with the supplied buffer, in a volume of $50 \mu \mathrm{l}$ containing $1.5 \mathrm{mmol} / 1 \mathrm{magnesium}$ chloride, $50 \mathrm{pmol}$ of each primer, $0.2 \mathrm{mmol} / \mathrm{l}$ of each dATP, dCTP, dGTP, and dTTP (Gibco-BRL), $5 \mu \mathrm{Ci} \alpha^{32} \mathrm{P}-$ $\mathrm{dATP}$, and $0.1 \mathrm{mg} / \mathrm{ml}$ BSA. Genomic amplification of exon 1 with a variety of primers led to non-specific amplification of spurious products even at raised temperatures. Because of this, genomic amplification of the $5^{\prime}$ portion of exon 1 was performed using the enzyme $P f u$ polymerase (Stratagene) in the supplied buffer in a total reaction volume of $100 \mu \mathrm{l}$, containing $50 \mathrm{pmol}$ of each primer, $0.2 \mathrm{mmol} / 1$ each of dATP, dCTP, dGTP, and dTTP, $5 \mu \mathrm{Ci} \alpha^{32} \mathrm{P}-$ dATP, and $10 \%$ dimethyl sulphoxide. For the $3^{\prime}$ end of exon 1 , and using a variety of primers and conditions, the correct product was present (as identified by blot hybridisation using a human probe), but constituted only a small fraction of the total reaction products.

Successful amplification and subsequent SSCP analysis of the $3^{\prime}$ end of exon 1 was restricted to RT-PCR alone. Poly-A ${ }^{+}$RNA was extracted from leucocytes or cultured fibroblasts using the QuickPrep Micro mRNA Purification Kit (Pharmacia Biotech). mRNA was reverse transcribed from gene specific cDNA synthesis primers using the enzyme Tth polymerase in the presence of manganese ions at temperatures between 65 and $70^{\circ} \mathrm{C}^{23}$ (fig $1 \mathrm{~A}$ ). These primers were designed to have $T_{m}$ values between 66 and $72^{\circ} \mathrm{C}$ so that reverse transcription could be carried out at raised temperatures (table). The first strand $\mathrm{CDNA}$ was then used as a template for PCR. All primers were designed manually as above. PCR was performed as before. For amplification of exon 1 cDNA, the addition of $10 \%$ dimethyl sulphoxide was again necessary to overcome problems caused by secondary structure within this $\mathrm{GC}$ rich region. 


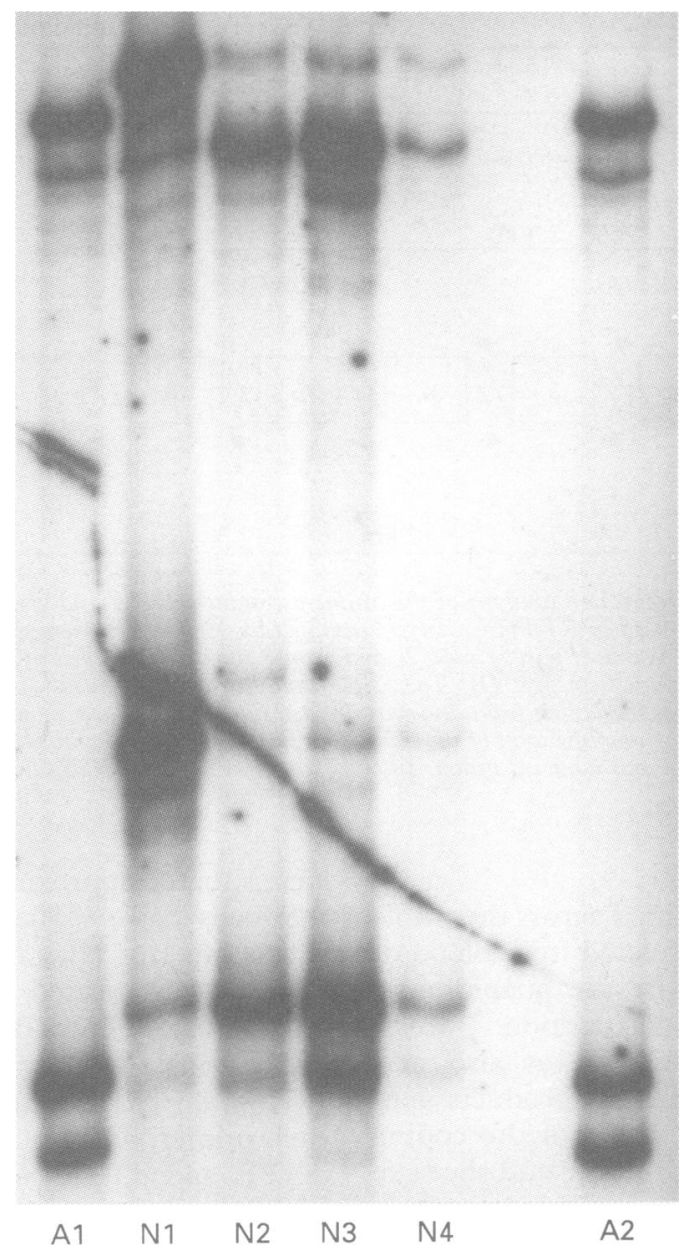

Figure 2 SSCP analysis of the $3^{\prime}$ end of exon 1 of two dogs diagnosed as deficient in $\alpha-L$-fucosidase ( $A 1$ and A2) and four normal animals (N1-N4). In all cases amplification was by RT-PCR.

\section{SEQUENCING}

PCR products were cloned into pCR-II using the TA Cloning System (Invitrogen) for sequence analysis. For the $3^{\prime}$ end of exon 1, products of approximately the expected size were purified by electrophoresis through agarose gels before cloning. Subcloned inserts were sequenced by the dideoxy chain termination method using the Sequenase Version 2.0 DNA Sequencing kit (Amersham/USB). In sequence analysis, the sequence of both strands was determined.

\section{Results}

The cloning and sequencing of the cDNA encoding canine $\alpha$-L-fucosidase has been described (Skelly et al, submitted for publication). The transcript encodes a polypeptide of 465 amino acids including a hydrophobic signal peptide, which is putatively cleaved from the mature protein. The percentage similarity between the canine and human forms of the enzyme at the cDNA level was found to be $84 \%$. The genomic structure of canine fucosidase was also determined and the introns partially sequenced. This information has been used to design intronic primers for amplification of exons for mutation analysis.
PCR products were generated for each of the exons $2-8$ using intronic primers. For each of these exons a single product was amplified which corresponded to the predicted size. SSCP analysis of these exons showed no differences between normal dogs, heterozygous dogs, and dogs known to have been homozygous for the fucosidosis allele (data not shown). Exon 1 was amplified in two parts. Difficulties were experienced in amplification of this exon, probably at least in part because of the G/C richness of both the exon and its surrounding introns. The $5^{\prime}$ end of the exon, when amplified using $P f u$ DNA polymerase and analysed by SSCP, showed no differences on PCR between normal, heterozygous, and homozygous mutant dogs. The $3^{\prime}$ end of the exon could not be amplified cleanly.

SSCP analysis was performed across the majority of the CDNA sequence using RT-PCR. This analysis of fibroblast and leucocyte $\mathrm{mRNA}$ from canine fucosidase normal and affected animals showed only one mobility shift, which was located in the $3^{\prime}$ portion of exon 1 (fig 2). This PCR product was cloned and sequenced in both normal and affected dogs and a 14 base pair deletion of the mRNA was identified at the $3^{\prime}$ terminus of the exon (fig $3 \mathrm{~A}$ ). This deletion causes a frameshift within the transcript and a premature stop codon is produced downstream in exon 2 . In the mutant cDNA, base 393 (EMBL accession number X92448) is now spliced to base 408 with a resultant frameshift in exon 2 of one nucleotide $5^{\prime}$. The mutant protein thus contains 25 novel amino acids before the first of two adjacent premature stop codons is encountered.

In order to determine the nature of this mutation in genomic DNA, amplification of the $3^{\prime}$ end of exon 1 was performed on genomic DNA from an affected animal using the primers described (table, fig 1B). As this PCR did not amplify with sufficient specificity, shotgun cloning of the size fractionated PCR products into pCR-II (Invitrogen) was necessary. Subsequent identification of the correct clone by sequence analysis allowed determination of the DNA sequence underlying the mutation. Surprisingly, this DNA also showed a deletion which was coextensive with that seen in the cDNA; that is, the final 14 base pairs of exon 1 were missing but the whole of intron 1 was present (fig 3B).

\section{Discussion}

In this paper we describe an alteration in the gene and transcript for the enzyme fucosidase in dogs suffering from fucosidosis. This deletion in the mRNA can be predicted to have major consequences in the subsequent translational processes because the frameshift induced by the deletion produces a premature termination codon. Hence it is predicted that the protein product would be foreshortened and consequently inactive and probably unstable. The absence of material cross reacting with antibodies raised against normal canine fucosidase in tissues of dogs suffering from fucosidosis could be because of protein in- 
A

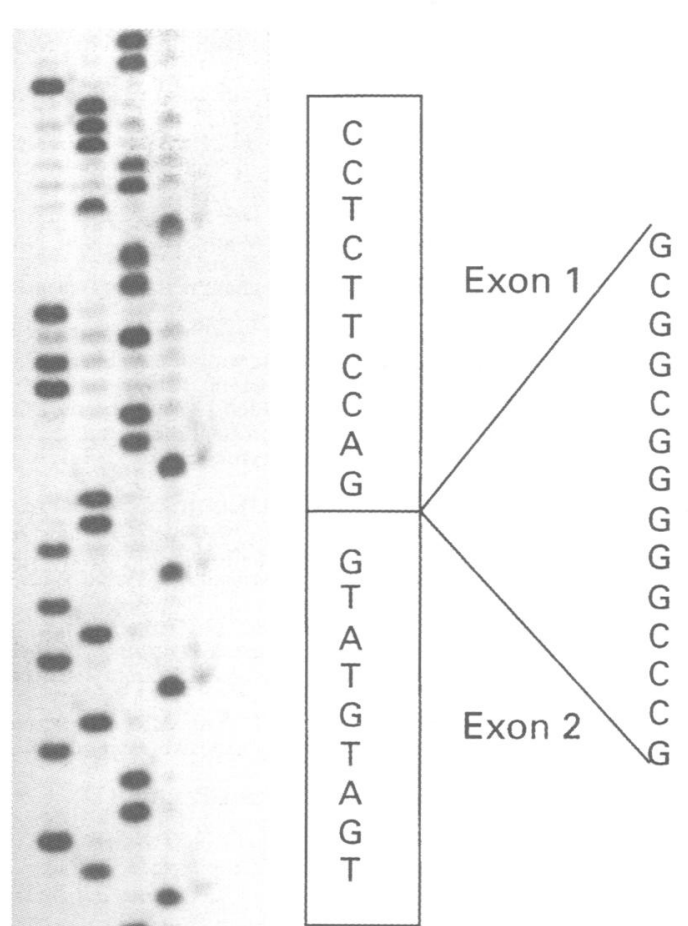

Normal sequence

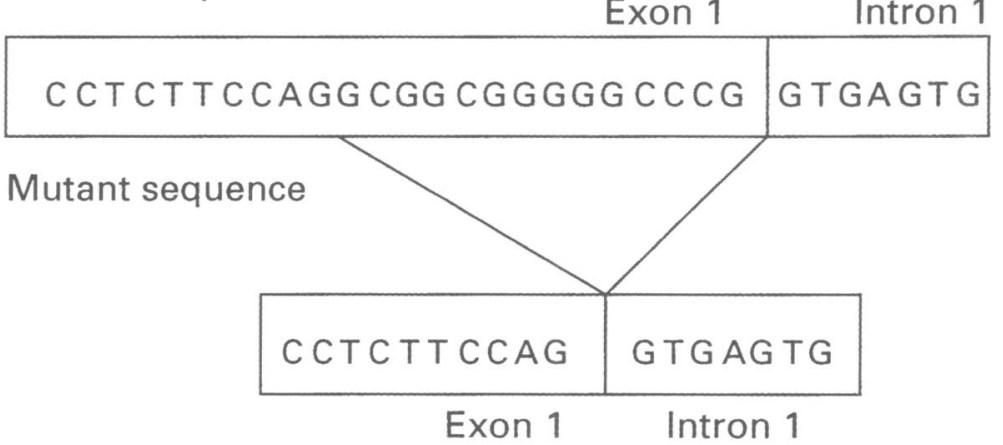

T G C A

Figure 3 (A) Sequence analysis of the $3^{\prime}$ end of exon 1 and $5^{\prime}$ end of exon 2 of canine fucosidase cDNA showing the 14 base pair cDNA deletion. (B) Comparison of mutant and normal sequences from genomic PCR products covering the $3^{\prime}$ end of exon 1 and $5^{\prime}$ end of intron 1 , showing the 14 base pair DNA deletion.

stability or of the absence of antigenic epitope in the mutant protein. ${ }^{24}$

The mutation underlying the deletion in the RNA is a coextensive deletion of the DNA. The splice donor site at the end of exon 1 in normal dogs takes the form "CCG/ GTGAGT..". This sequence, with a $\mathrm{C}$ at position -2 , is itself less close to a splice donor consensus than the splice site created by the deletion in the mutant gene "CAG/ GTGAGT..". It is therefore unsurprising that the latter splice site is used efficiently.

The description of the disease causing mutation has important consequences for the English springer spaniel breed. Until now, heterozygotes have been identified on the basis of a reduced enzyme activity. ${ }^{8}$ This method is unreliable and far from satisfactory when breeding programmes are being established. The description of the mutation at the molecular level will allow a test to be designed which will identify the mutant allele specifically, so that heterozygous dogs can be identified easily and rapidly.

Fucosidosis in English springer spaniels provides a useful animal model with which to study the human disease. Dogs diagnosed biochemically as being homozygous for the diseasecausing mutation have already been used in trials to assess the efficacy of bone marrow transplantation. ${ }^{72526}$ As a consequence of this work, bone marrow transplantation has been attempted in a human patient. ${ }^{27}$ Now, with the therapeutic emphasis turning towards the possibility of gene replacement therapy, a fuller molecular understanding of the canine disease must be beneficial. Preliminary assessments of gene transfer, using retroviral vectors, have been conducted in human and canine fucosidosis fibroblast cultures. ${ }^{28}$ The early identification of affected animals and the knowledge of the cDNA and genomic organisation of canine fucosidase could prove invaluable when it comes to designing such gene replacement protocols using the springer spaniel as a human disease model.

We would like to thank Helen Cragg for her advice and tremendous encouragement in the earlier stages of this project, also David Entz who provided technical support. This work was funded by the Wellcome Trust. BJS is the holder of a Wellcome Clinical Research Studentship.

1 Durand P, Barrone C, Della Cella G. Fucosidosis. F Pediatr 1969;75:665-74.

Hartley WJ, Canfield PJ, Donnelly TM. A suspected new canine storage disease. Acta Neuropathol 1982;56:225-32.

3 Littlewood JD, Herrtage ME, Palmer AC. Neuronal storage disease in English springer spaniels. Vet Record 1983;112:
86-7.

4 Abraham D, Blakemore WF, Dell A, et al. The enzymic defect and storage products in canine fucosidosis. Biochem f 1984;221:25-33. 5 Herrtage ME. Canine fucosidosis. Vet Annual 1988;28:223-
7 . 
6 Ferrara ML, Taylor RM, Stewart GJ. Age at marrow transplantation is critical for successful treatment of canine plantation is critical for successful treatment

7 Taylor RM, Farrow BRH, Stewart GJ, Healy PJ, Tiver K. The clinical effects of lysosomal enzyme replacement by
. bone marrow transplantation after total lymphoid irradiation on neurologic disease in fucosidase deficient dogs. Transplant Proc 1988;20:89-93.

8 Barker CG, Herrtage ME, Shanahan F, Winchester BG. Fucosidosis in English springer spaniels: results of a trial screening programme. $\mathcal{f}$ Small Anim Pract 1988;29:62330.

9 Occhiodoro T, Beckman KR, Morris CP, Hopwood JJ. Human $\alpha$-L-fucosidase: complete coding sequence from cDNA clones. Biochem Biophys Res Commun 1989;164: 439-45.

10 Kretz KA, Cripe D, Carson GS, Fukushima H, O'Brien JS. Structure and sequence of the human $\alpha$-L-fucosidase gene Structure and sequence of the human $\alpha$-L-fucosi
and pseudogene. Genomics 1992;12:276-80.

11 Willems PJ, Darby JK, DiCioccio R, et al. Identification of a mutation in the structural alpha-L-fucosidase gene in a mutation in the structural alpha-L-fucosida

12 Kretz KA, Darby JK, Willems PJ, O'Brien JS. Characterisation of the EcoRI mutation in fucosidosis patients: a stop codon in the open reading frame. $\mathcal{F} \mathrm{Mol}$ Neurosci 1989;1:177-8

13 Willems PJ, Gatti R, Darby JK, et al. Fucosidosis revisited: a study of 77 patients. Am f Med Genet 1991;38:111-31.

14 Williamson M, Cragg H, Grant J, et al. A $5^{\prime}$ splice site mutation in fucosidosis. $\mathcal{f}$ Med Genet 1993;30:218-23.

15 Seo HC, Willems PJ, Kretz KA, Martin BM, O'Brien JS. Fucosidosis: four new mutations and a polymorphism. Hum Mol Genet 1993;2:423-29.

16 Seo HC, Willems PJ, O'Brien JS. Six additional mutations in fucosidosis: three nonsense mutations and three frameshift fucosidosis: three nonsense mutations and the
mutations. Hum Mol Genet 1993;2:1205-8.

17 Seo HC, Kunze J, Willems PJ, et al. A single base deletion in a Turkish patient with fucosidosis. Hum Mutat 1994; 3:407-8
18 Seo HC, Yang M, Tonlorenz R, et al. A missense mutation (S63L) in $\alpha$-L-fucosidase is responsible for fucosidosis in an Italian patient. Hum Mol Genet 1994;3:2065-6.

19 Seo HC, Heidemann PH, Lutz E, O'Brien JS. A nonsense mutation in two German patients with fucosidosis. Hum Mutat 1995;6:184-5.

20 Jeanpierre $M$. A rapid method of purification of DNA from blood. Nucleic Acids Res 1987;15:9611.

21 Orita M, Iwahana H, Kanazawa H, Hayashi K, Sekiya T. Detection of polymorphisms of human DNA by gel electrophoresis as single strand conformation morphisms. Proc Natl Acad Sci USA 1989;86:2766-70.

22 Orita M, Suzuki Y, Sekiya T, Hayashi K. A rapid and sensitive detection of point mutations and genetic polymorphisms using the polymerase chain reaction. Genomics 1989;5:874-9.

23 Myers TW, Gelfand DH. Reverse transcription and DNA amplification by a Thermus thermophilus DNA poly-

24 Barker C, Dell A, Rogers M, Alhadeff JA, Winchester BG Canine $\alpha$-L-fucosidase in relation to the enzymic defect and storage products in canine fucosidosis. Biochem $f$ 1988;254:861-8.

25 Taylor RM, Farrow BRH, Stewart GJ. Correction of enzym deficiency by allogenic bone marrow transplantation following total lymphoid irradiation in dogs with lysosomal storage disease (fucosidosis). Transplant Proc 1986;18:326 9.

26 Taylor RM, Farrow BRH, Stewart GJ. Amelioration of clinical disease following bone marrow transplantation in fucosidase-deficient dogs. Am $\mathcal{F}$ Med Genet 1992;42: 628-32.

27 Vellodi A, Cragg H, Winchester B, et al. Allogeneic bone marrow transplantation for fucosidosis. Bone Marrow marrow transplantation

28 Occhiodoro T, Hopwood JJ, Morris CP, Anson DS. Correction of $\alpha$-L-fucosidase deficiency in fucosidosis fibroblasts by retroviral vector-mediated gene transfer. Hum Gene Ther 1992;3:365-9. 